\title{
Editorial
}

\section{Preguntas para pensar las nuevas realidades educativas}

\author{
María Elena Pease Dreibelbis ${ }^{1}$ \\ Universidad Científica del Sur \\ mpease@cientifica.edu.pe
}

Igor Valderrama Maguiña²

Universidad Científica del Sur

ivalderrama@cientifica.edu.pe

ORCID: https://orcid.org/0000-0002-0016-1934

\section{María Angélica Pease Dreibelbis ${ }^{3}$ \\ Pontificia Universidad Católica del Perú \\ mapease@pucp.edu.pe \\ ORCID: https://orcid.org/0000-0003-2645-4580}

Citar como: Pease, M. E., Valderrama, I. y Pease, M. (2021). Preguntas para pensar las nuevas realidades educativas. Desde el Sur, 13(2), e0015.

Si algo nos ha dejado en claro la pandemia producto de la covid-19, es la profunda desigualdad que configura a nuestro país. El sistema educativo no es la excepción. La experiencia de participación del sistema educativo - tanto en la educación básica como en la superior-, desde el inicio del aislamiento social, se ha visto visiblemente condicionada por el contexto en el cual se encuentran aprendices y docentes, su acceso a la conectividad y el conjunto

\footnotetext{
1 Magíster en Cognición, Aprendizaje y Desarrollo (Pontificia Universidad Católica del Perú) y bachiller en Artes Plásticas (PUCP). Docente de posgrado en la Universidad Científica del Sur y la Universidad Antonio Ruiz de Montoya, especializada en currículo, didáctica y formación docente. Asesora pedagógica en colegios y para el programa Aprendo en casa de la Unidad de Arte y Cultura del Minedu. Es miembro del Grupo de Investigación en Psicología, Cultura y Género.

2 Magíster en Docencia para la Educación Superior por la Universidad Andrés Bello y licenciado en Filosofía por la PUCP, con estudios de Psicoanálisis en la Nueva Escuela Lacaniana (NEL-Lima). Ha sido consultor en temas de formación docente y diseño curricular (Unesco) e investigador asociado de la UARM. Se desempeñó como especialista del Minedu, encargado del diseño y validación de pruebas estandarizadas para docentes. Es docente y asesor de tesis de posgrado.

3 Ph. D en Psicología y magíster en Psicología Cognitiva por la Universidad de Columbia (Nueva York). Licenciada en Antropología por la PUCP, es profesora principal del Departamento de Psicología de la PUCP, coordinadora del proyecto "Ser adolescente en el Perú» (Unicef-PUCP y miembro del Grupo de investigación en Psicología, Cultura y Género. Es investigadora en temas de psicología adolescente, diversidad y género y actriz y directora teatral, miembro del grupo /Otro/colectivo teatro.
} 
de recursos materiales e inmateriales que les sirven de soporte, que abarcan desde la gestión hasta el apoyo socioemocional.

Ir a la escuela o a la universidad ha implicado experiencias muy dispares que van desde participar de sesiones sincrónicas a través de plataformas virtuales durante largas jornadas, oyendo a un docente explicar diapositivas, hasta ver televisión, escuchar breves programas radiales por altavoz en los centros comunitarios para luego realizar un trabajo, tomarle una foto y enviarla por el celular a un docente.

Todos los niveles y modalidades educativas se han visto afectados por la emergencia sanitaria; sin embargo, sabemos poco sobre el impacto de esta diversidad de experiencias en los aprendices, sus familias y la sociedad. Sabemos poco de las lecciones, confusiones y vacíos que están dejando en nuestros aprendices. Preocupa, sin embargo, mucho. Preocupa que las brechas de todo tipo —urbana/rural, varón/mujer, privado/público- se abran más y generen desigualdades de mayor complejidad. Preocupa que las experiencias de aquellos que transitaron a un nuevo sistema (por ejemplo, que salieron de la secundaria y entraron a la universidad o instituto superior) sean particularmente más complicadas, al sumarse a los retos de dichas transiciones. Preocupa que aumenten las tasas de deserción y dejen truncas tantas trayectorias. En medio de todas estas transformaciones, que demandan desde la academia respuestas y evidencias, asistimos además a una nueva: el proceso de retorno a clases que, dada la eficiencia del proceso de vacunación, es ya una realidad. Los nuevos desafíos y estrategias nos retan a plantear nuevas preguntas. 
Las preguntas que necesitamos hacer a la realidad educativa en este momento, sin duda, no se agotan en los trabajos que se comparten en esta edición, pero constituyen un primer esfuerzo de reflexión en torno al tema, que tiene la ventaja de efectuarse sobre la misma marcha de los acontecimientos.

Podríamos organizar las preocupaciones y reflexiones que este contexto nos demanda en algunos ejes que articulan preguntas e inquietudes, los cuales no son necesariamente excluyentes, sino que se conectan e interactúan. De un lado, se hace necesario identificar el impacto de la pandemia en nuestra tan diversa realidad; en particular, en cuanto a aquellos ámbitos o dimensiones que ya eran críticos antes de la pandemia, como las condiciones socioculturales, económicas e institucionales de nuestra sociedad. ¿Qué nuevos retos debemos atender en la inclusión, en sentido amplio, la equidad de género, socioeconómica, etc.? ¿Cómo se han visto no solo evidenciadas sino ampliadas las brechas educativas, de género, tecnológicas, territoriales y culturales? ¿Cómo han contribuido los diversos tipos de apoyo del Estado y de las instituciones a la continuidad educativa (chips telefónicos, tablets, datos, entre otros) y qué nos dicen los beneficiarios sobre este elemento como diferenciador en su educación? ¿Se necesitará sostener?

Es imprescindible preguntarnos quiénes y cómo han accedido a las diversas propuestas educativas, cómo han sido sus experiencias y qué han logrado, para plantear soluciones desde las distintas realidades sociales, económicas, culturales y educativas, así como desde las representaciones de los actores mismos sobre los problemas y las alternativas de solución. 
Otro eje articulador gira en torno a un aspecto central de la educación: los aprendizajes. En términos de resultados, ¿qué aprendizajes se lograron y cuáles se postergaron en 2020 para un 2021 que sigue acumulando rezagos? Los egresados de nuestras universidades, ¿habrán logrado las habilidades necesarias para los retos que se les presenten en el futuro? La necesaria flexibilización y priorización de algunas competencias sobre otras, ¿cómo impacta en los planes curriculares y en la consolidación de aprendizajes? Se requiere elaborar diagnósticos de los aprendizajes reales, vigentes, que permitan subsanar brechas y atender las nuevas necesidades para revisar los planes de estudios de acuerdo con esta nueva realidad.

Los aprendizajes integran dimensiones cognitivas y socioemocionales; el proceso de aprendizaje se desarrolla a partir de nuestras propias referencias culturales y nuestros conocimientos previos, sobre los cuales se construye el nuevo. Este proceso demanda múltiples oportunidades para dicha construcción, a fin de ensayar versiones y formulaciones propias y hacerlas dialogar tanto con pares como con otras generaciones, para luego reflexionar hasta consolidar nuestros nuevos conocimientos y nuestro posicionamiento frente a otras perspectivas. Así también, demanda múltiples interacciones, ya sea con docentes, pares y recursos diversos dentro de los cuales nuestro entorno, la acción, el mundo real ofrecen las mejores condiciones para desarrollar aprendizajes significativos y profundos. De ahí la relevancia en observar qué oportunidades de aprendizaje han sido privilegiadas durante estos años pandémicos y qué interacciones se han logrado establecer y potenciar a través de los formatos, plataformas y diversos medios para el aprendizaje a los cuales los estudiantes han tenido acceso. 
Los modelos pedagógicos en las escuelas y universidades que ya se encontraban en tránsito hacia modelos por competencias y el desarrollo de las competencias para el siglo XXI (Unesco y Orealc, 2017) se vieron retados por la necesidad de atender la educación a distancia durante la emergencia sanitaria. La reacción inicial llevó a trasladar el modelo presencial, contenidos y actividades, al entorno virtual, pero este modelo no se mantuvo estático. Las instituciones educativas fueron generando cambios y mejoras en el camino. Por ello, cabe preguntarse en qué medida se han visto afectados los modelos pedagógicos. ¿Se ha logrado un proceso de innovación o se han consolidado formas tradicionales de entender la educación, pero ahora bajo un rostro tecnológico? Sabemos que mayor información no ofrece obligatoriamente mayores oportunidades de aprendizaje o condiciones para el desarrollo de la autonomía académica del aprendiz; los entornos educativos estandarizados no atienden a la complejidad de contextos y capacidades de los estudiantes. En ese sentido y desde la contraparte docente, ¿se ha priorizado la formación de capacidades docentes centrada en cursos aislados o se han desarrollado competencias digitales que transformarán la experiencia educativa en adelante?

Nos preguntamos, entonces, ¿aprender con el uso de la tecnología es una opción o un imperativo? ¿Es la educación a distancia una forma de democratizar la educación? Nos preocupan algunos aspectos de la universidad que ya eran problemáticos o que implican un desafío para la gestión universitaria: bajo qué ejes de calidad se está pensando la educación virtual, dada la predominancia de paradigmas del cambio educativo que tienden a considerar que lo tecnológico per se permite la transformación de los aprendizajes. Esto implica modos de inversión y de gestión centrados en el diseño de entornos virtuales o tecnológicos novedosos pero que no ofrecen reales oportunidades 
de aprendizaje. La enseñanza remota de emergencia ${ }^{4}(\mathrm{Hod}-$ ges et al., 2020) no es lo mismo que educación en línea, cuyas modalidades, diseños instruccionales, roles de los participantes (instructores, docentes, tutores, estudiantes) y medios de evaluación y retroalimentación son variables que organizar en el modelo elegido. La enseñanza remota de emergencia es más limitada y cabe preguntarse por el caso peruano, ¿cuánto ha integrado en sus propuestas las experiencias de los estudiantes? ¿Cuáles han sido los diversos roles de la docencia en este proceso y bajo qué condiciones? Así, se trata de analizar si la práctica institucional existente con sus posibilidades y limitaciones perpetúa una forma tradicional de operar o si brinda nuevas y reales posibilidades de aprendizaje a los estudiantes, además de ofrecer condiciones de calidad para que los docentes y el conjunto de recursos universitarios cumplan metas consistentes.

De acuerdo con el Currículo Nacional vigente, «educar es acompañar a una persona en el proceso de generar estructuras propias internas, cognitivas y socioemocionales, para que logre el máximo de sus potencialidades» (Minedu, 2016, p. 11). ¿Los docentes están preparados para realizar este acompañamiento durante estas condiciones extraordinarias? ¿Quién apoya a los docentes? Esto nos presenta un último eje que nos interpela directamente y que es fuente de enorme preocupación; tiene que ver con el impacto del aislamiento social y del vivir en un contexto de pandemia sobre el sentido de bienestar y salud mental de nuestros estudiantes. Sabemos que nos enfrentamos a múltiples duelos. Muchos hemos perdido a personas significativas, de manera relativamente súbita y sin poder participar del soporte social y de los diversos rituales

4 ERT por las siglas en inglés de emergency remote teaching. 
que nos alivian el dolor de dichas pérdidas. Nos preocupa imaginar los múltiples duelos que han afectado a nuestros estudiantes, el tipo de contención que han recibido, la manera como viene transformándose su visión sobre la vida y el fin de esta, los nuevos temores que emergen y el acompañamiento que han podido tener para afrontar esas dificultades.

La escuela cumple funciones que van mucho más allá del logro de aprendizajes. Constituye un espacio crucial de socialización con los pares, de interacción con adultos fuera del entorno familiar, de acceso a recursos y fuentes de soporte diverso. Este rol se vuelve más importante a medida que el estudiante tenga mayor grado de exclusión económica o de acceso a otros recursos. En ese sentido, el impacto que ha generado el no participar de la escuela y de todos estos recursos es sin duda una de las grandes preguntas que desde la investigación convendría ir empezando a abordar.

Los estudiantes universitarios, especialmente en sus primeras etapas, requieren no solo de un espacio académico de formación, sino también de soporte para consolidar una identidad universitaria. Esto ya era un reto en el contexto prepandemia. La modalidad presencial conlleva un marco de interacciones sociales que permiten al estudiante ser parte de una vida universitaria que fortalece su motivación, amplía sus posibilidades de aprendizaje y le permite configurar redes académicas y profesionales: interacción con sus pares y docentes dentro y fuera del aula y el establecimiento de comunidades de interés alrededor de temas académicos o profesionales. Esta mirada integral del desarrollo universitario no está consolidada en las propuestas educativas peruanas. ¿Cómo se ha remarcado esta situación en el contexto virtual? ¿Qué nuevas condiciones y retos genera la virtualidad que, según venimos oyendo, 
ha venido para quedarse a algún nivel en la educación superior?

De manera complementaria, es necesario estudiar también el impacto en el campo docente. El súbito tránsito a la educación a distancia por consecuencia de la pandemia, en un sistema que no estaba preparado para ello, ha sido, seguramente, uno de los retos más grandes que la mayoría de docentes de nuestro país ha enfrentado. Los horarios de trabajo y condiciones laborales se han visto trastocados, las demandas de aprendizaje se incrementaron y la sobrecarga se ha hecho visible. El doble rol de cuidado de hijos y acompañamiento en labores escolares al mismo tiempo que trabajar desde casa ha supuesto, además, retos complejos y dificultades cuyo impacto aún no terminamos de mapear. Pero, asimismo, los docentes también han sufrido pérdidas de seres queridos, también enfrentan duelos y también han participado de un contexto de incertidumbre e imprevisibilidad. La manera como la pandemia ha impactado sus vidas constituye, de igual modo, un tema en agenda por ser investigado.

Si miramos históricamente, desigualdades institucionalizadas y fórmulas de cambio que no llegan con la suficiente rapidez, no podemos dejar de ver la situación como una oportunidad. Las múltiples y esforzadas iniciativas por atender las brechas de acceso y calidad de la educación han sido insuficientes. Desde distintos ámbitos de la academia y la sociedad se viene pensando cómo reimaginar la educación, y desde esa tarea queremos contribuir a pensar cómo formular nuevas formas de entender ese espacio-tiempo que es el eje central del proceso pedagógico; cómo seleccionar y articular el contenido y sus múltiples interacciones, para desarrollar tanto lo individual como lo colectivo; cuáles y cómo pueden potenciarse los diversos acompañamientos que necesitamos las personas en 
cada etapa de nuestro desarrollo para reconocernos como aprendices permanentes, personas que piensan y actúan en la sociedad para el bien común, actual y futuro. ¿Cuántas pandemias necesitamos para darle un nuevo rumbo, justo y sostenido, a la vida educativa de las personas? Esperamos contribuir a detonar con estos temas y pensar qué se necesita estudiar, indagar, profundizar, para así ir con paso firme por rutas que ofrezcan alternativas según nuestras diversas realidades.

La presente edición de Desde el Sur constituye un esfuerzo desarrollado con la urgencia que supone este momento, de compartir preguntas y generar evidencias que permitan abrir y ampliar rutas para estas preocupaciones. 


\section{REFERENCIAS BIBLIOGRÁFICAS}

Banco Interamericano de Desarrollo (BID). (2020). Perú: Desafíos del desarrollo en el post COVID-19. Septiembre 2020. https://publications.iadb. org/publications/spanish/document/Peru-Desafios-del-desarrollo-en-elpost-COVID-19.pdf

Consejo Nacional de Educación (CNE). (2020). Proyecto Educativo Nacional. PEN-2036. El reto de la ciudadanía plena. https://www.cne.gob.pe/uploads/ publicaciones/2020/proyecto-educativo-nacional-al-2036.pdf

Hodges, C., Moore, S., Lockee, B., Trust, T. y Bond, M. A. (2020). The difference between emergency remote teaching and online learning. Educause Review. https://er.educause.edu/articles/2020/3/the-difference-betweenemergency-remote-teaching-and-online-learning

Ministerio de Educación del Perú (Minedu). (2016). Currículo Nacional de la Educación Básica. http://www.minedu.gob.pe/curriculo/

Naciones Unidas. (2020). La educación en tiempos de la pandemia de COVID-19. https://repositorio.cepal.org/bitstream/handle/11362/45904/ S2000510_es.pdf?sequence $=1$ \&isAllowed $=y$

Unesco-Orealc. (2017). Reporte: Educación y habilidades para el siglo XXI. Reunión Regional de Ministros de Educación de América Latina y el Caribe, Buenos Aires, Argentina, 24 y 25 de enero 2017. Orealc/Unesco Santiago). http://www.unesco.org/new/fileadmin/MULTIMEDIA/FIELD/ Santiago/pdf/Informe-Reunion-Buenos-Aires-2017-E2030-ALC-ESP.pdf 